\title{
From the CSIRO
}

\section{Sight-saving science: specialist eye care using satellite technology}

Patients living in rural and remote areas have little or no access to regular eye screening. Eye conditions such as diabetic retinopathy can lead to vision loss or blindness if not detected early and treated.

To overcome this problem, the CSIRO has developed a low-cost system that uses broadband satellite technology to screen people living in remote and rural areas who are at risk of eye disease.

A special low-cost camera is used to capture full high-resolution images of a patient's retina. The encrypted images are then securely forwarded via a broadband connection to a city-based ophthalmologist who examines them, using a system called Remote-I.

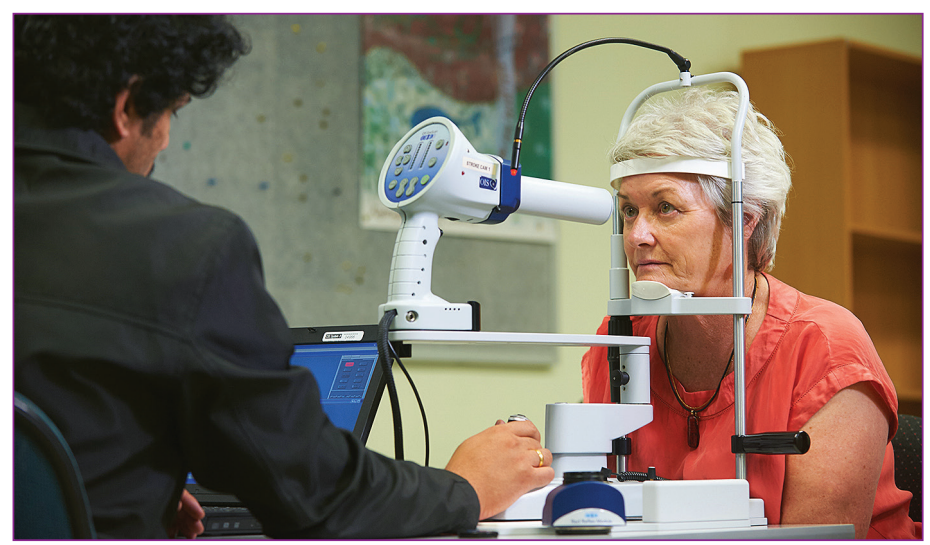

Reproduced with permission
More than 1100 patients - mainly Indigenous and older Australians - have now participated in trials conducted in conjunction with health authorities in Western Australia and Queensland. Satellite dishes were installed at trial sites, mainly at local medical clinics for the trials, and local nurses were trained to take the retinal photos.

Over the 1-year trial period:

- 82 patients were diagnosed as having diabetic retinopathy (DR) two of them were diagnosed with proliferative DR and another two with severe non-prolific DR;

- 63 patients were diagnosed as having macular oedema;

- more than $80 \%$ of the participants screened had no eye problems, reducing pressure on specialists and improving service efficiency.

The outcomes of this study show that the patients and health providers were highly satisfied with the tele-eye care consultations over broadband satellite; speed of eye related data transmission was adequate; and clinic cost savings were achieved.

This research project is the first to investigate and report on the practical delivery of satellite-based telehealth services to remote and rural areas in Australia. It has improved access to eye care for residents of the trial sites and shown that routine eye examination could enable early detection of sight-threatening eye diseases. Most importantly, this type of service could prevent vision loss.

Yogesan Kanagasingam 